\title{
Gestión del conocimiento a través de plataformas y herramientas digitales de aprendizaje ante la migración de clases presenciales a en línea
}

\section{Knowledge management through digital learning platforms and tools due to migration face-to-face classes to online}

María Guadalupe Morales-Espíndola'; Karla Coré Moreno-Cortés²; María Martha del Socorro Romano-Cadena3; María del Refugio García-Alarcón ${ }^{4}$

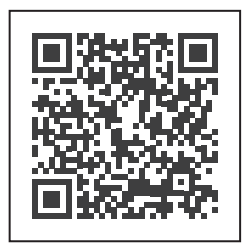

Palabras clave: innovación, plataformas digitales de aprendizaje, gestión del conocimiento, TIC, COVID-19.

Articulo de investigación:

Fecha de recepción:

24/05/2020

Fecha de aceptación:

14/08/2020

Esta publicación se encuentra bajo licencia:

Creative Commons

Reconocimiento-

NoComercial-

SinObraDerivada 4.0

Internacional

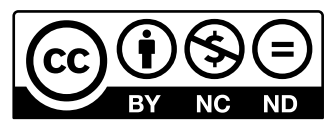

\section{Resumen}

El presente artículo trata el tema de la gestión de las tecnologías de la educación en ambientes de aprendizaje virtuales. Ante un evento histórico, como lo es la pandemia de la COVID-19, el sistema educativo se vio en la necesidad de migrar clases presenciales en educación a distancia. Este hecho, que tomó por sorpresa a maestros y estudiantes, ha generado diversas áreas de oportunidad para mejorar las habilidades en el uso de las TIC tanto en estudiantes como en profesores. Es por ello por lo que el objetivo de este artículo es recomendar estrategias para gestionar adecuadamente el conocimiento ante la necesidad de migrar de clases

1 Licenciada en Administración Pública y Ciencias Políticas. Doctora en Investigación Educativa. Profesora investigadora, Benemérita Universidad Autónoma de Puebla, México. gualumespindola@hotmail.com, ORCID: https://orcid.org/0000-0002-1579-6318

2 Licenciada en Administración de Hoteles y Restaurantes. Doctora en Investigación Educativa. Profesora investigadora, Benemérita Universidad Autónoma de Puebla, México. karla.morenoco@correo.buap.mx, ORCID: https://orcid.org/0000-0001-5673-2428

3 Licenciada en Administración de Empresas. Doctora en Investigación Educativa. Profesora investigadora, Benemérita Universidad Autónoma de Puebla, México. martha.romano@correo.buap.mx, ORCID: https://orcid. org/0000-0002-2402-0933

4 Licenciada en Derecho. Magíster en Administración y Gestión de Instituciones Educativas. Magíster en Ciencias de la Educación. Profesora investigadora, Benemérita Universidad Autónoma de Puebla, México.smary_2003buap@ hotmail.com, ORCID: https://orcid.org/0000-0002-2184-3838 


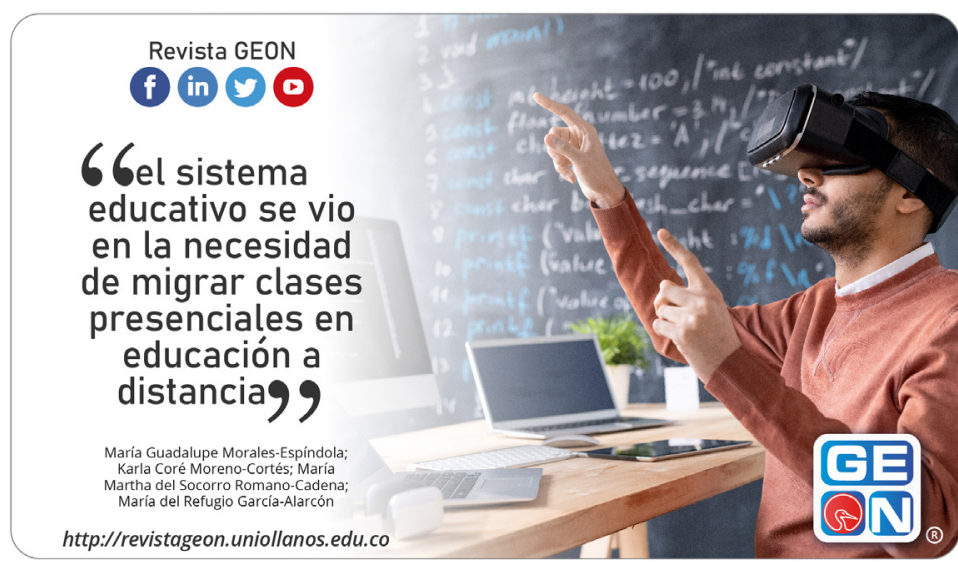

presenciales a ambientes cien por ciento en línea en el nivel superior, cuando existan situaciones de emergencia que impidan el aprendizaje tradicional, a través del diagnóstico de la experiencia vivida en la pandemia de la COVID-19.

La investigación realizada se centró en estudiantes de la Facultad de Administración de la Benemérita Universidad Autónoma de Puebla y la respuesta de estos ante los retos educativos que les representa el uso de plataformas digitales para dar continuidad a sus cursos presenciales.

La metodología utilizada se basó en un análisis experimental-cualitativo, con apoyo de la técnica de los grupos focales, para ahondar en las diversas perspectivas y opiniones de los sujetos de estudio que hicieron una migración obligatoria a una edu-

Cómo citar este artículo / Toreference this article:

Morales Espíndola, M. G., Moreno Cortés, K. C., Romano Cadena, M. M. del S., \& García Alarcón, M. del R. (2020). Gestión del conocimiento, a través de plataformas y herramientas digitales de aprendizaje ante la migración de clases presenciales a en linea. Revista GEON (Gestión, Organizaciones Y Negocios), 7(2), 1-19. https://doi. org/10.22579/23463910.217 cación en línea.

Los resultados demuestran áreas de oportunidad para mejorar la gestión del aprendizaje en ambientes digitales.

Palabras clave: innovación, plataformas digitales de aprendizaje, gestión del conocimiento, TIC, COVID-19.

Códigos JEL: A22 General Economics and Teaching 
Cómo citar este artículo / Toreference this article:

Morales Espíndola, M. G., Moreno Cortés, K. C., Romano Cadena, M. M. del S., \& García Alarcón, M. del R. (2020). Gestión del conocimiento, a través de plataformas y herramientas digitales de aprendizaje ante la migración de clases presenciales a en linea. Revista GEON (Gestión, Organizaciones Y Negocios), 7(2), 1-19. https://doi. org/10.22579/23463910.217

\section{Abstract}

This article deals with the issue of managing educational technologies in virtual learning environments. Faced with a historical event, such as the COVID-19 pandemic, the educational system was forced to migrate face-to-face classes in distance education.

This fact, which took teachers and students by surprise, has generated various opportunity areas to improve skills, by using ICT in students and teachers. That is why the objective of this article is to recommend strategies to adequately manage knowledge in the face of the need to migrate from face-to-face classes to 100 percent on line at the college/university level, when there are emergency situations that force to change traditional learning, through of the diagnosis of the experience lived in the COVID-19 pandemic.

The research carried out focused on students from the Faculty of Administration of the Benemérita Universidad Autónoma de Puebla and their response to the educational challenges posed using digital platforms to continue their classroom courses.

The methodology used was based on an experimental-qualitative analysis, with the support of the focus group technique, which allows us to understand the diverse perspectives, opinions and even feelings that was generate in the participants of this study, who have had to migrate from education traditional to a virtual one.

The results demonstrate multiple opportunity areas to improve learning management, through innovative techniques, but without neglecting the structural differences that exist between face-to-face and distance education.

Keywords: Innovation; Knowledge management; ICT; Digital learning platforms; COVID-19. 


\section{Introducción}

El uso de las tecnologías de la información y la comunicación (TIC) en el ámbito educativo no es un tema nuevo. Se han llevado a cabo innumerables estudios acerca de los beneficios de la aplicación de las tecnologías y de la importancia de estas para generar mejores competencias a nivel profesional. Resulta evidente que el sistema educativo, en especial la educación superior, ha hecho esfuerzos para que tanto alumnos como docentes incorporen herramientas de aprendizaje que fortalezcan sus habilidades en el uso de las tecnologías mencionadas.

En el caso específico de la Benemérita Universidad Autónoma de Puebla (BUAP), se enlistan como competencias genéricas (es decir, que todo estudiante del nivel superior debe tener) el desarrollo de habilidades en el uso de las tecnologías de la información y comunicación (DHTIC), y se espera que el profesionista logre gestionar la información, las tecnologías y los procesos de comunicación para fortalecer la formación personal y profesional a través de las TIC, al ser utilizados en trabajos académicos y científicos de manera ética, creativa y asertiva (Benemérita Universidad Autónoma de Puebla, 2010).

\section{Problemática encontrada}

Como es sabido, a finales del año 2019, en la provincia de Wuhan, en China, empezaron a presentarse casos masivos de una nueva enfermedad denominada por la Organización
Mundial de la Salud como COVID-19. El virus causante de esta enfermedad, altamente contagioso, pronto trascendió fronteras y se convirtió en pandemia. El primer caso de COVID-19 en México se registró el día 27 de febrero de 2020 y a partir del día 17 de marzo la BUAP se sumó a la suspensión total de clases, como medida de precaución para evitar la propagación del virus en México (Milenio Digital, 2020).

A partir de esta situación, tanto maestros como estudiantes se enfrentaron al reto de hacer una migración de cursos a plataformas digitales, todo ello con el interés de mantener el aprendizaje y seguir con el plan de estudios de cada materia, a pesar de no poder salir de casa y ante la ventaja de contar con una gran diversidad de herramientas digitales educativas en la actualidad.

Sin embargo, esta migración ha presentado un reto para todos, puesto que a pesar de que algunos profesores ya implementaban ejercicios utilizando herramientas digitales, el hecho de sustituir al cien por ciento materias presenciales por materias en línea no es sencillo; es evidente que se requieren conocimientos específicos, disciplina y, por supuesto, contar con herramientas como internet y teléfono inteligente, tableta o computadora, para poder dar seguimiento a todas las actividades.

En México, el 74\% de las zonas urbanas cuentan con servicio de internet, mientras que, en las zonas rurales, la cifra es del $40,6 \%$, y aunque existen 
74,3 millones de usuarios de internet, solo el 45\% de los hogares cuentan con una computadora (Secretaría de Comunicaciones y Transporte, 2019).

Ante esto, vale la pena conocer qué son las plataformas virtuales de aprendizaje, cuál es su función, qué herramientas digitales se integran en ellas, cuáles son sus ventajas y desventajas y cuántas clases existen.

\section{Objetivo}

Recomendar estrategias para gestionar adecuadamente el conocimiento ante la necesidad de migrar de clases presenciales a ambientes cien por ciento en línea en el nivel superior, cuando existan situaciones de emergencia que impidan el aprendizaje tradicional, a través del diagnóstico de la experiencia vivida en la pandemia de la COVID-19.

\section{Contexto teórico}

\section{Plataformas LMS}

Una plataforma LMS es un espacio virtual de aprendizaje. LMS es un acrónimo que en inglés significa Learning Management System. Estas plataformas permiten la creación y gestión de cursos completos para internet sin necesidad de tener conocimientos profundos de programación o de diseño gráfico. Se manejan un conjunto de herramientas agrupadas y optimizadas para fines docentes. La formación académica de los profesores es importante para el proceso de adquisición de habilidades y destrezas necesarias para aplicar críticamente todos los recursos tecnológicos disponibles en esta época y para generar nuevos modelos de comunicación educativa que favorezcan la innovación docente (Lemus Pool, 2012).

Las plataformas virtuales de aprendizaje, conocidas como sistemas de gestión de aprendizaje, funcionan como un grupo de aplicaciones en un entorno cliente-servidor, donde del lado del servidor se instala un software especial que controla todas las operaciones internas, para garantizar una operación eficiente, y del lado del cliente, los usuarios del sistema utilizan un navegador y herramientas complementarias a la computadora (tales como elementos audiovisuales y accesorios internos), con la finalidad de realizar un proceso didáctico de manera no presencial (por medio de internet) y de gestionar la retroalimentación entre el profesor y los demás compañeros de clase (ParadisoLMS, 2020).

Entre las principales funciones de las plataformas virtuales de aprendizaje se encuentran: a) comunicar a todos los usuarios o integrantes de la clase con los profesores; b) gestionar el acceso interno al sistema de los usuarios, cada uno de ellos con su clave y contraseña de acuerdo con su perfil (profesor, alumno, administrador, director, entre otros); c) realizar evaluaciones a los alumnos según los parámetros y exigencias de cada materia o clase; d) gestionar los recursos digitales de los usuarios y las clases (videos, libros virtuales, evaluaciones, archivos de texto, bases de datos, 
entre otros); e) gestionar servicios o módulos para permitir la interacción entre sus usuarios, siendo los más comunes las sesiones de videoconferencia, chats, foros y mensajería instantánea; f) creación de informes de las evaluaciones realizadas, el consumo de recursos, la elaboración de correcciones, actualizaciones al sistema, entre otros; y g) las plataformas virtuales de aprendizaje ofrecen una serie de herramientas muy valiosas que permiten al docente utilizar las que considera convenientes a fin de crear ambientes de aprendizaje significativo que le permitan cumplir los objetivos de su curso.

Existen diversas clases de plataformas: plataformas propietarias, de código abierto y de desarrollo propio (Universidad Santander Panamá, 2018).

\section{Plataformas propietarias}

Se debe pagar por ellas para tener derecho a la instalación y el mantenimiento. Su valor está en función del número de usuarios, por lo general, su pago es anual y para renovar se debe pagar nuevamente. Incluyen herramientas y aplicaciones muy completas y complejas que permiten una mayor facilidad en el seguimiento de un curso virtual. Algunas de estas son: First Class, WebCT, eCollege (a Person company) y Blackboard.

\section{Plataformas de código abierto}

Aunque el software libre no es sinónimo de gratuidad, sino de libertad, suelen ser gratuitas. Ofrecen un tipo especial de licencia, Ilamada GPL (licencia pública general), que posibilita cuatro libertades a los usuarios: 1. La libertad de usar el programa con cualquier propósito. 2. La libertad de estudiar cómo funciona el programa y adaptarlo a las necesidades, esto es, la libertad de acceder al código fuente como una condición previa para esto. 3. La libertad de distribuir copias del programa. 4. La libertad de mejorar el programa y hacer públicas las mejoras a los demás, de modo que toda la comunidad se beneficie. Ejemplos de estas son: Claroline, Dokeos, Ilias, Moodle, entre otras.

\section{Plataformas de desarrollo propio}

Este tipo de plataformas se diferencia de las dos anteriores porque no están dirigidas a su comercialización, como las de venta, ni pretende una distribución masiva a un conjunto de organizaciones, como las de software libre. Surgen en instituciones y grupos de investigación, con el objetivo de responder a necesidades educativas concretas.

\section{Materiales y métodos}

La presente investigación es de carácter exploratorio y con métodos cualitativos, sustentado en la técnica de investigación denominada focus group debido a que estos permiten adentrarse en la dinámica interna de los sujetos de estudio y profundizar así en sus sentimientos, actitudes, creencias, motivaciones y en todo aquello que subyace en su conducta Hernández, 2014). 
Con el apoyo de estudiantes de la materia Investigación de Mercados (alumnos de la BUAP), se llevaron a cabo 10 focus group con la participación de 8 a 10 integrantes cada uno. Todos los participantes fueron alumnos activos de la Facultad de Administración de la BUAP y se encontraban recibiendo clases virtuales (4 semanas mínimo) durante el desarrollo de la investigación.

Debido a las medidas de prevención ante la pandemia de la COVID-19, los focus groups fueron realizados mediante la plataforma Discord, en la semana del 20 al 26 de abril de 2020, con una duración promedio de 60 minutos.

Los criterios de selección de los participantes fueron los siguientes: 1. Que no se conocieran entre sí (es decir, que fueran de distintas licenciaturas y semestres). 2. Que estuvieran inscritos en la modalidad presencial en primavera 2020 y, por tanto, hubiesen "vivido la migración forzosa" a clases virtuales. 3. Que no fueran familiares de los alumnos que se encontraban moderando el focus group. 4. Que contaran con una computadora, tableta o teléfono, internet y la disponibilidad para participar en la investigación. Se contó con la participación de un total de 98 estudiantes, 66 mujeres y 32 hombres, todos ellos estudiantes de la Facultad de Administración de la BUAP, de las licenciaturas en Gastronomía, Administración de Empresas, Turismo y Comercio Internacional.

Tal como lo propone Morgan (2018), se utilizó como instrumento de apoyo al moderador, una guía de entrevista, en la cual se explicaron las normas de participación, los agradecimientos por el tiempo invertido y el carácter académico del focus group. Para iniciar, se realizaron cuatro preguntas (denominadas de calentamiento o rompehielos), cuya función fue generar confianza entre el moderador y los participantes y, posteriormente, se hicieron preguntas correspondientes al tema de interés.

\section{Preguntas de calentamiento:}

1. ¿Qué tan adecuado consideran que es su equipo electrónico y su conexión a internet?

2. ¿Cuánto tiempo aproximadamente utilizas este equipo?

3. ¿Cuentan con las habilidades suficientes para manejarlos?

4. ¿Qué tan acostumbrados están a realizar trabajos y tareas utilizando estos equipos electrónicos?

5. ¿Sus profesores les permiten utilizar regularmente estos equipos en las clases presenciales?

\section{Preguntas del tema de interés:}

1. ¿Qué métodos o estrategias han utilizado sus profesores para continuar con las clases y el aprendizaje durante esta situación?

2. ¿Qué ventajas han tenido las clases virtuales para ti?

3. A la hora de tomar una clase en cualquier plataforma, ¿existen barreras de comunicación que les afectan? ¿Cuáles? 
4. ¿Presentan alguna dificultad en el uso de las diferentes plataformas que se están utilizando durante esta situación?

5. ¿Hubo cambios de horario en sus clases o mantuvieron su horario?

6. ¿Sienten que las clases virtuales son una buena alternativa para continuar sus cursos ante esta situación?

7. ¿Cómo calificas la calidad de tu aprendizaje en las clases virtuales, comparado con las clases presenciales?

8. ¿Consideras que la cantidad de trabajos y tareas actualmente es similar a la que se tenía en las clases presenciales?

9. ¿Te sientes más presionado en las clases presenciales o en las virtuales?

10. ¿Has tenido problemas para entrar (o entregar actividades) a tus clases virtuales? ¿Cómo han reaccionado tus profesores?

11.¿Cómo ha afectado el avance de tus aprendizajes esta migración a plataformas y clases virtuales?

12.¿Qué propuestas harían a sus profesores para mejorar o hacer más eficientes las clases virtuales?

Conclusión de la entrevista:

- Cuál es tu opinión sobre la sesión realizada el día de hoy y si tienes algún otro comentario para concluir nuestra investigación, por favor, coméntalo.
- Gracias a todos ustedes por su participación en la sesión y por los valiosos aportes que nos brindaron frente a la investigación que estamos realizando.

- Igualmente les damos las gracias por el tiempo que nos han dedicado y que esperamos haya sido de su agrado esta sesión.

\section{Resultados}

Respecto a las preguntas de calentamiento o rompehielos, los datos más llamativos fueron: el 75\% de los entrevistados consideraron inadecuado o incompleto el equipo electrónico con el que cuentan para recibir clases virtuales. Un 90\% reportó que tuvieron problemas con la conectividad a internet en varias ocasiones y que tanto ellos como sus profesores tuvieron que suspender las clases por estos inconvenientes. Se mencionó como situación común el hecho de que el $60 \%$ de los estudiantes foráneos, al regresar a sus lugares de origen, no contaban con internet y tuvieron que asistir a lugares públicos para lograr conectividad o, en su caso, cafés-internet.

Solo el 20\% de los participantes en el estudio cuenta con computadora propia. Los que la tienen llevan en promedio 3 años con su equipo. Los demás alumnos comparten la computadora con sus hermanos o padres y un $50 \%$ de los participantes utiliza su teléfono inteligente como única herramienta de apoyo ante las clases virtuales. 
El $100 \%$ de los alumnos consideran que cuentan con los conocimientos necesarios y las habilidades suficientes para manejar las plataformas virtuales.

Únicamente un 30\% de los estudiantes estaba acostumbrado a utilizar plataformas digitales de aprendizaje para enviar tareas o realizar trabajos y exámenes en las clases presenciales. El 85\% tenía grupos de comunicación como Facebook o WhatsApp con sus profesores, siendo estas las principales herramientas virtuales con las que se trabajaba antes de la pandemia.

En la figura 1 se muestran los resultados con respecto a las herramientas más utilizadas.

Figura 1. Gráfica de las herramientas digitales más utilizadas durante la pandemia, año 2020.

Herramientas más utilizadas

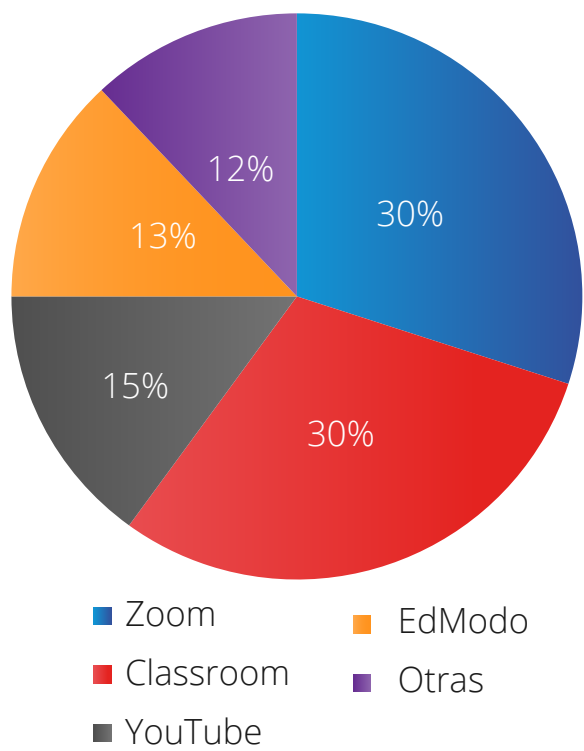

Fuente: elaboración propia.
Solo el 5\% de los profesores utilizaron las plataformas de aprendizaje como Blackboard o Moodle (oficiales de la BUAP y las cuales se usan en forma regular para impartir cursos en las licenciaturas semipresenciales o a distancia). El motivo que mencionaron los docentes para no utilizar estas herramientas fue que desconocían sus funciones o que eran más complicadas que otras herramientas.

Respecto a las ventajas de las clases en línea, un 55\% de los estudiantes valoran el hecho de que gracias a estas no perdieron el semestre y continuaron con sus cursos; sin embargo, un 45\% mencionó que no veían ventajas en este tipo de estrategias, ya que no sentían impacto real en su aprendizaje, se distraían demasiado o no entendían las indicaciones de sus profesores.

En la figura 2 se muestran las principales barreras de comunicación mencionadas.

Un 75\% de los participantes en el focus group han tenido dificultades con las herramientas digitales. La más mencionada es Zoom, cuyo principal problema es "sacarlos de las sesiones" o "voz cortada y falta de calidad de la imagen". Un 35\% de los alumnos reporta falta de flexibilidad por parte de algunos docentes, los cuales no los apoyaron cuando tuvieron problemas técnicos con las plataformas o herramientas. Un dato preocupante fue que solo un $50 \%$ de sus docentes se interesaron en hacer la migración en línea durante las dos primeras semanas de confinamiento por la COVID-19; el resto se comunicó con ellos 
después de 15 días y un 10\% no se había comunicado ni dejado trabajos hasta el momento del estudio.

Figura 2. Principales barreras de comunicación durante el desarrollo de las clases virtuales, año 2020.

Principales barreras de comunicación

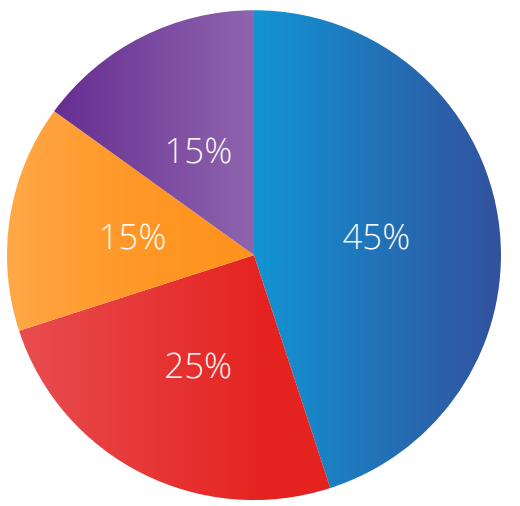

10

- Desidia por parte de los profesores

- Baja calidad de internet

- Reglas de evaluación poco claras e incertidumbre por su calificación

- Falta de interés del alumno)

Fuente: elaboración propia.

El 88\% de los participantes considera que la "educación en línea o a distancia" que reciben es deficiente, ya que solo un 30\% de los profesores les dan "clases en tiempo real o les graban tutoriales". La mayoría solo les dejó lecturas y cuestionarios y no les explicaron los objetivos de dichas actividades. En la figura 3 se observan las materias más mencionadas por su alto grado de dificultad para ser comprensibles a distancia.

El 100\% de los entrevistados coinciden en que sienten más estrés y pre- sión en las clases virtuales y que la calidad de su aprendizaje no es comparable con los estudios presenciales.

Figura 3. Materias consideradas complicadas de comprender durante la migración en línea, año 2020.

Materias con alto grado de dificultad para ser comprendidas durante la migración virtual

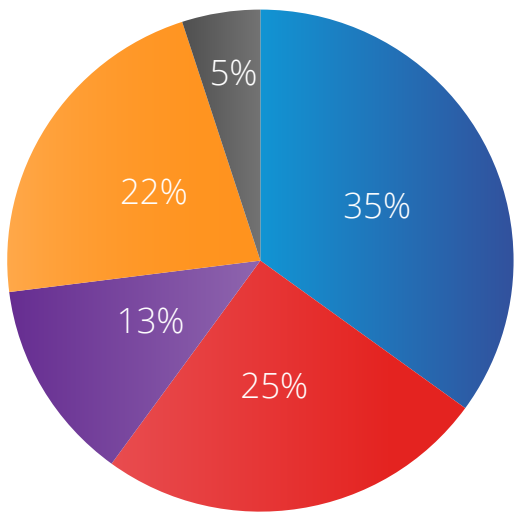

- Estadística

- Contabilidad

- MatemáticasFinancieras

- Laboratorios de alimentos

- Otras

Fuente: elaboración propia.

\section{Discusión}

Ante los resultados obtenidos a partir de la investigación, es evidente que la migración de clases presenciales a clases en línea representó un reto muy grande tanto para estudiantes como para docentes.

Es por ello por lo que se debe aprender de esta experiencia y generar innovación mediante la implementación de herramientas que permitan 
la gestión del conocimiento, a través del uso de la TIC, considerando que esto conduce tanto a maestros como alumnos a desarrollar y utilizar las competencias y habilidades para crear un proceso de enseñanza en el que se fortalezca el aprendizaje significativo.

La gestión del conocimiento, tema de importancia en este artículo, va más allá de transmitir información a los alumnos. Tal como lo destacan Nonaka y Takeuchi, el conocimiento es el proceso humano y dinámico de justificar las creencias personales en busca de la verdad, es decir, gestionar el conocimiento implica una toma de conciencia del valor del conocimiento como recurso y producto en la sociedad (Ordóñez, 2001).

El conocimiento es el tesoro más preciado que pueda tenerse y buscarse. La información se convierte a través de los individuos en un activo de conocimiento para la organización y este, a su vez, en un activo de capital humano (Arceo, 2009).

La pandemia por COVID-19 obliga a incorporar de manera permanente el uso de las TIC en espacios educativos, para propiciar la gestión del conocimiento a través de experiencias innovadoras que muestran la forma en que los nuevos entornos virtuales de aprendizaje pueden ser útiles en las aulas de clase, es decir, no solamente se trata de transmitir información, sino de generar análisis y fortalecer el desarrollo profesional del individuo, por lo que mediante la adecuada gestión del conocimiento se puede gene- rar una amplia gama de aplicaciones y procedimientos tales como aprendizaje basado en la red y la computadora, plataformas y herramientas digitales, información vía internet, extranet, intranet, audio y video.

A pesar de que existen innumerables herramientas, plataformas, tutoriales y cursos, la pandemia puso al descubierto la falta de pericia para migrar una clase presencial en una virtual.

Resulta evidente que la educación en línea requiere de un cambio de paradigma, en el cual, los estudiantes deban ser más disciplinados y autodidactas y los profesores más flexibles, creativos y hábiles en el manejo de las TIC. Esta pandemia muestra rezagos importantes en materia educativa y, peor aún, diferencias sociales muy marcadas, que inciden en el desempeño y gestión del conocimiento de los estudiantes. Basta recalcar que una de las problemáticas que mencionaron los sujetos de estudio de la presente investigación es que no contaban con un equipo propio y que debían compartirlo con sus padres y hermanos, ya que algunos debían hacer home office y tareas de diferentes niveles educativos. Así también debe considerarse que esta migración de lo presencial a en línea tomó por sorpresa a la mayoría de la población; por tanto, no existieron condiciones idóneas, aunado a la situación psicológica que permeó el ánimo de la sociedad y que hizo complicado el adaptarse ante una situación inédita, no solo para México, sino para el mundo, como lo fue la pandemia por COVID-19. 
A partir de los resultados obtenidos y respondiendo al objetivo general del presente estudio, el cual fue recomendar estrategias para gestionar adecuadamente el conocimiento ante la necesidad de migrar clases presenciales a ambientes cien por ciento virtuales cuando existan situaciones de emergencia que impidan el aprendizaje tradicional, a través del diagnóstico de la experiencia vivida en la pandemia de la COVID-19.

Es importante recalcar que, en primer lugar, los docentes deben comunicar a sus estudiantes los criterios "adaptados" de evaluación ante clases virtuales, los cuales no pueden ser los mismos que los presenciales. Algunos participantes del estudio consideraban, por ejemplo, que los exámenes a distancia resultan mucho más sencillos, ya que pueden tener a la mano sus apuntes o ayudarse en "equipo"; por tanto, esta estrategia de evaluación debe combinarse con muchas otras, como lo son portafolios de evidencias, cuadros sinópticos, resúmenes, mapas mentales, participación en foros, resolución de casos de estudio, creación de contenido como wikis, juegos virtuales, blogs, entre otros.

En segundo lugar, es deseable que las universidades aprovechen al máximo las plataformas digitales de aprendizaje si es que cuentan con ellas, ya que representan una inversión importante. Si bien es cierto que en el marco de las licenciaturas denominadas presenciales como parte del tronco común se imparte la materia Desarrollo de Habilidades para las
Tecnologías de la Información y la Comunicación, aún falta un largo camino para que tanto estudiantes como docentes aprovechen al máximo todos los beneficios de estas tecnologías.

Así mismo, en la BUAP (universidad en donde se llevó a cabo la investigación) existen innumerables cursos que se ofertan para que los docentes se capaciten en el uso de plataformas como Blackboard y Moodle (que son con las que oficialmente se trabaja en la universidad). De igual forma, existe una constante preocupación por aumentar la participación tanto de docentes como alumnos en estas plataformas y con la implementación de programas sustentables con el ambiente incluso hay sugerencias de usarlas en conjunto con herramientas digitales en clases presenciales como medios para revisar tareas y hacer exámenes en computadora.

A pesar de ello, en este estudio se evidenció que Blackboard y Moodle no fueron plataformas digitales utilizadas y aprovechadas en todo su potencial. Es muy importante fomentar más cursos de capacitación para alumnos y profesores y facilitar el uso de estas plataformas en la educación presencial, especialmente porque aún existe incertidumbre mundial acerca de los "cambios de vida" que representará para todos la COVID-19. No es seguro que se regrese en una fecha en específico, ni se conocen las condiciones, por lo cual es altamente probable que tanto la educación presencial como los trabajos requieran adaptarse y manejar, al menos en un porcentaje, clases a distancia y 
uso del home office. La pandemia nos ha enfrentado a una nueva realidad y hay que adaptarse.

Algo de suma importancia es comprender que la educación en línea no es cien por ciento responsabilidad del maestro y tampoco del estudiante. El profesor requiere interactuar con sus alumnos, resolver dudas, grabar tutoriales, retroalimentar sus trabajos, entre otros; esto representa mucho trabajo y gestionar y planear el conocimiento. Los docentes están trabajando más horas, pero no es válido solo enviar tareas o lecturas; en esta era en donde la información sobra, el desde casa, y cada profesor debe ser el "facilitador" que guíe y elija los materiales, herramientas digitales y estrategias más adecuadas para que sus alumnos aprendan. Así también los alumnos deben enfrentarse a un cambio en donde sean más responsables de su gestión del conocimiento, deban aprender a manejar sus tiempos, evitar en lo posible las distracciones propias del hogar y construir criterios e ideas que permitan un aprendizaje colaborativo y constructivista.

De igual forma es importante recalcar que se requiere apertura, positivismo y paciencia para afrontar una migración a la educación a distancia. En realidad, todos tienen el reto de re-aprender; el proceso no es sencillo y no será rápido. Cabe remarcar que gracias a la internet y a la infinidad de herramientas con las que se cuenta en la actualidad han sido posibles avances impresionantes. Para no ir más lejos, en otras condiciones o épocas, ante un evento como la COVID-19, hubiese resultado imposible continuar trabajando y aprendiendo desde casa, y si bien es cierto que existen innumerables aspectos susceptibles de mejorar, debemos comprender que es posible cambiar, aportar y mejorar para lograr avances significativos en el uso de las tecnologías aplicadas a la educación. A causa de la pandemia se han abierto oportunidades de cursos, conferencias y pláticas a distancia que permiten abrir el panorama, acercar las distancias y entender que el futuro es ahora. Hoy el maestro enfrenta en el aula las consecuencias de un mundo cambiante a causa de la revolución que ha generado la globalización, la tecnología y las comunicaciones. Por tanto, deberá promover el pensamiento creativo y crítico de los contenidos curriculares y culturales, combinar la teoría y la práctica, así como la formación en valores. Por consiguiente, el docente deberá establecer políticas, estrategias de enseñanza y de evaluación para lograr la formación integral de los estudiantes. La nueva forma de educar requiere docentes con competencias profesionales y con un rol distinto en el proceso de enseñanza-aprendizaje, con un conjunto de conocimientos que le permitan educar a las nuevas generaciones de profesionales.

Es complicado determinar el perfil y las competencias del docente porque hay que definir la educación que se requiere hoy para formar a los profesionales del futuro y definir el proceso de enseñanza considerando los contenidos que impartirá. De esa forma se determinarán los cursos que 
debe adquirir. La actualización le capacitará para el ejercicio profesional como docente y estará preparado para enfrentar los retos que el presente y el futuro le deparan (Arias \& Arias, 2018).

En la actualidad, el profesor debe innovar y aprovechar las facilidades y ventajas de las TIC:

[...] esta herramienta ofrece interactividad, comunicación, dinamismo en la presentación de contenidos, uso de multimedia, texto y elementos que permiten atender a los usuarios con distintos estilos de aprendizaje, todo en un mismo sitio: la computadora con conexión a la red.

Esta fuente de inagotables servicios ha sido abrazada por algunos educadores como un recurso para la enseñanza y por algunas instituciones educativas como el sistema que les permite ampliar sus aulas sin tener que levantar nuevas paredes (Scagnoli, 2001).

En consecuencia, el perfil del docente lo conforma un conjunto de competencias que integran conocimientos, destrezas, habilidades, aptitudes, actitudes y valores, los cuales pondrá en práctica en el salón de clase para enseñar a los estudiantes a construir sus conocimientos y a desarrollar las competencias que aplicará en el ejercicio profesional.

En un artículo de investigación de la revista Killkana Sociales de Ecuador (2019), realizado sobre las compe- tencias profesionales del docente universitario desde una perspectiva integral, se enmarca teórica y conceptualmente el tema y se describen los hallazgos del estudio sobre el comportamiento del indicador tecnología dentro de las competencias personales, el cual fue designado por los docentes consultados con el más bajo puntaje en cuanto al resto de los indicadores (dominio de la disciplina, formación pedagógica y capacidad de realización); sin embargo, se ubica como suficiente de acuerdo con el rango establecido.

El resultado manifiesta que, en la institución estudiada (Instituto Universitario de Tecnología), el uso de la tecnología por parte del personal docente no constituye una actividad fundamental; es decir, se evidencia poca valoración hacia el uso y aplicación de la tecnología, más aún al desarrollo tecnológico, lo cual se considera una debilidad extrema por tratarse de una organización cuya misión y visión están orientadas, precisamente, a la tecnología. En este contexto, el comportamiento demostrado por la tecnología se aleja del planteamiento de Carnoy, Castells, Cohen y Cardoso (1997) cuando sostienen que el gran desarrollo de la ciencia y la tecnología contemporáneas, cuya manifestación más evidente y acelerada es el creciente proceso de informatización de la sociedad, debe reflejarse en el docente universitario en su explotación pertinente a través del uso de las nuevas tecnologías de la información y la comunicación, en función de su labor docente como herramienta insustituible (Hinestroza, Silva, \& Iturralde, 2019). 
El presente contexto y las tendencias actuales en la educación obligan a replantear las herramientas y los métodos de enseñanza que las universidades y profesores deben utilizar. Representa un paradigma pedagógico conceptualizado y orientado por nuevas estrategias de enseñanza-aprendizaje con alternativas que conducen al manejo dinámico del tiempo y del espacio para dar cumplimiento a la misión institucional y a los objetivos académicos. Se plantea una educación apoyada en ambientes virtuales de aprendizaje (AVA). Para ello, será necesario contar con una capacitación integral del docente, enfocada al dominio de las herramientas virtuales de la enseñanza y el aprendizaje que le permita tener encuentros sincrónicos o asincrónicos con sus estudiantes y centrarse en el educando, considerando el qué y el cómo realizará su proceso.

El aula virtual debe ser diseñada de modo que los alumnos tengan la posibilidad de ser expuestos a situaciones similares de práctica del conocimiento, pero, sobre todo, a una retroalimentación que les permita conocer cuál es el grado de aprovechamiento que van adquiriendo en cada temática.

Como aportación para futuras investigaciones en esta área, es importante que se revisen aspectos tales como: ¿qué tipo de acceso tendrá al aula virtual, si será limitado o tendrá todos los derechos?; ¿quién realizará la actualización y monitoreo del sitio?; ¿cuál será la capacidad para el archivo de materiales y el tiempo que los mate- riales estarán en línea para el acceso? Como recomendación en futuras investigaciones, se considera oportuno consultar el punto de vista de los docentes y de los mismos estudiantes cuando ambos valoren, después de la "vuelta a la nueva normalidad", los beneficios de la educación a distancia. Incluso es deseable aplicar un nuevo focus group con exactamente las mismas preguntas y sujetos de estudio, pero una vez que estos hayan tenido más semanas de clases a distancia (cabe recordar que este estudio se realizó cuando en México se llevaban 30 días de confinamiento); sin embargo, las percepciones y los sentimientos pueden modificarse conforme los días pasan e incluso cuando se regrese a las clases presenciales.

\section{Conclusiones}

Debido al período de cuarentena por el que se transita a nivel mundial, a causa de la pandemia por COVID-19, las instituciones educativas de nivel básico, medio superior y superior tanto públicas como privadas han hecho uso de las plataformas y herramientas digitales de aprendizaje para continuar el trabajo de forma efectiva a pesar de no estar totalmente preparados. De acuerdo con los resultados que se obtuvieron a partir de la investigación, algunos docentes y alumnos están familiarizados con su uso; sin embargo, la gran mayoría normalmente imparten sus clases de forma presencial en aulas físicas usando esporádicamente herramientas y plataformas digitales como complemento de sus cursos. 
Esta investigación buscó: 1. Enlistar las plataformas y herramientas utilizadas por profesores y alumnos ante la migración virtual. 2. Analizar las ventajas y desventajas de diversas plataformas adaptadas y adoptadas por los profesores y alumnos para lograr el aprendizaje virtual. 3. Indagar sobre los sentimientos de los estudiantes ante el aprendizaje en línea y explorar tanto las bondades que encuentran en la nueva forma de estudiar como las desventajas o problemáticas que han detectado. 4. Comparar el nivel de aprendizaje que los estudiantes obtienen de las clases presenciales vs. las clases online. Y finalmente 5. Medir la percepción de los estudiantes respecto a las estrategias que sus profesores emplearon ante esta emergencia y conocer su opinión sobre la efectividad en el logro de sus aprendizajes significativos. Lo anterior con el objetivo de proponer una metodología de innovación y gestión del conocimiento para facilitar la migración de clases presenciales a virtuales, especialmente porque, como la historia lo ha demostrado, esta clase de pandemias no terminan en meses, se requieren años y una adaptación a nuevas realidades y formas de vida.

Se puede hablar de beneficios que tienen estudiantes y profesores con el uso de las plataformas y herramientas digitales: a) se cuenta con recursos ilimitados debido a su formato digital, a diferencia de una clase tradicional; b) se reducen los costos de trasporte para los usuarios y el tiempo que ello implica, así como gastos en papelería, documentación, entre otros; c) se cuenta con mayor facilidad de acceso para los usuarios del sistema y se puede personalizar cada entorno; d) hay un registro de toda la información de manera digital y está disponible en cualquier hora del día, además de que se pueden establecer restricciones en los tiempos de entrega y recepción de trabajos, así como exámenes autocalificables; e) existe la posibilidad de conectarse desde cualquier tipo de dispositivo móvil: celulares, tabletas, laptops, computadoras de escritorio, entre otros; f) la comunicación suele ser más personalizada; g) otorga la posibilidad de continuar con los estudios desde diversas partes del mundo y a los docentes les permite continuar trabajando y percibiendo un salario.

Sin embargo, después de la investigación realizada, se enumeran las desventajas; entre ellas: a) mayor esfuerzo y dedicación por parte del profesor; en varias ocasiones se personalizan las dudas y se requiere una inversión importante de tiempo para migrar materiales didácticos que se usaban en las clases presenciales y adaptarlos a las plataformas en línea; asimismo, los estudiantes requieren que se les graben tutoriales, se les facilite el acceso a lecturas y ejercicios de alto valor y se resuman los contenidos; b) el acceso a los medios y la brecha informáticos implica que el estudiante deba disponer permanentemente de los medios informáticos. En México existe un rezago importante sobre conectividad de alta calidad a internet y una gran parte de la población comparte con sus familiares 
equipos como computadoras o tabletas, dividiéndose así los tiempos de uso entre varios integrantes de una familia; c) no todos los docentes cuentan con los conocimientos básicos de informática necesarios para usar una plataforma virtual. Por tanto, deben invertir tiempo en ver tutoriales e investigar o practicar para lograr conocer y manejar estas herramientas; d) la metodología de autoestudio en la educación en línea exige al estudiante un mayor esfuerzo y disciplina que en clases presenciales. Al respecto, aún falta mucho camino por recorrer para lograr que los alumnos se vean a sí mismos como protagonistas de su propio aprendizaje. En pleno 2020, muchas clases se siguen impartiendo basadas en el aprendizaje conductista $y$, por consiguiente, resulta complicada la migración a una educación más abierta y flexible, como lo es la educación a distancia; e) contacto directo: los estudiantes y maestros acostumbrados a las clases presenciales presentan una gran dificultad cuando se trabaja de forma totalmente digital. Se puede generar una sensación de soledad y aislamiento y la retroalimentación por parte del profesor no es inmediata, lo que puede provocar desmotivación del estudiante o incluso un "alejamiento" que genera poca interacción social e incluso depresión (Vivas, 2020).

\section{Referencias}

Arias, M., Arias, E. A., Arias, J., Ortiz M. M., \& Garza M.(2018, junio). Perfil y competencias del docente universitario recomendados por la UNES-
CO y la OCDE. Revista Atlante: Cuadernos de Educación y Desarrollo. https://www.eumed.net/rev/atlante/2018/06/competencias-docente-universitario

Anuario Estadístico Institucional 20162017. (2017). Benemérita Universidad Autonóma de Puebla. https:// bit.ly/2QHtnaY

Criterios y niveles de dominio de las competencias genéricas de la BUAP. (2010). Benemérita Universidad Autónoma de Puebla. https://bit. ly/3bic2ug

27 plataformas virtuales educativas gratuitas. (2020, 7 de abril). Cengage. https://bit.ly/2EOQLRv

Díaz, V. (2019, 30 de septiembre). Generación de leads: todo lo que debes saber. Neo Attack. https://neoattack.com/generacion-de-leads/

Hernández, R. F. (2014). Metodología de la Investigación (6.a ed.). McGraw-Hill Education.

Hinestroza, M., Silva, M., Iturralde, S. \& Maderos, M. (2019). Competencias profesionales del docente universitario desde una perspectiva integral. Revista Killkana Sociales, 3(1), 1-14. https://doi.org/10.26871/killkana_social. v3i1.443

Lemus-Pool, M. C. (2012). El desarrollo de las habilidades digitales en profesores universitarios [Tesis, Universidad Autónoma de México]. https://bit.ly/ 31FLOyk

Kreuger, R. A. \& Casey, M. A. (2000) Focus Groups: A Practical Guide for Applied Research (3.a ed.). Sage Publications. 
Méndez, F., \& Mendoza, D. (2018, 14 de enero). México ocupa cuarto lugar a nivel mundial en el uso de las redes sociales. UNAM Global. http://www. unamglobal.unam. $m \times / ? p=31030$

Caída del peso y suspensión de clases: cronología del coronavirus en México. (2020, 29 de marzo). Milenio. https://www.milenio.com/politica/coronavirus-mexico-linea-mapa-resumen-covid19

Morgan, C. (2018). Using the Focus Group in Market Research. B2B International. https://www.b2binternational.com/ publications/market-research-focus-group/

Onwuegbuzie, A. J., Dickinson, W. B., Leech, N. L. \& Zoran, A. G. (2017). Un marco cualitativo para la recolección y análisis de datos en la investigación basada en grupos focales. Paradigmas: Una Revista Disciplinar de Investigación, 3(2), 127-57. https://dialnet.unirioja. es/servlet/articulo?codigo=3798215

Ordóñez, D. P. (2001). La gestión del conocimiento como base para el logro de una ventaja competitiva sostenible la organización occidental vs la japonesa. Investigaciones Europeas de Dirección y Economía de la Empresa, 7(3), 91-108.

¿Qué son las plataformas virtuales de aprendizaje? (2020, 4 de abril). Paradiso Solutions. https://www.paradisosolutions.com/es/plataformas-virtuales-aprendizaje

Peña, K., Pérez, M., \& Rondón, E. (2010). Redes sociales en Internet: reflexiones sobre sus posibilidades para el aprendizaje cooperativo y colabirativo. Revista de Teoría y Didáctica de las Ciencias Sociales, 173-205.

Romano, M., García, A. \& Martínez, M. (2017, 20-30 de abril). Experiencia
B-Learning y el uso de la TIC en Facultad de Administración y Unidad Regional Atlixco de la BUAP y ENSFEP. Eje temático 3: Blended learning: Experiencias en busca de la calidad [sesión de conferencia]. EduQ@2017 - VII Congreso Virtual Iberoamericano de Calidad en Educación Virtual y a Distancia, Buenos Aires, Argentina.

Ruiz de Querol, R. (2017). La sociedad de la informática. UOC.

Scagnoli, N. (2001). El aula virtual: usos y elementos que la componen. https:// bit.ly/32HfHCy

Schwartzman, G., Tarasow, F., \& Trech, M. (2012, 1-3 de febrero). La educación en línea a través de diversos dispositivos tecno-pedagógicos [sesión de conferencia]. III Congreso Europeo de Tecnologías de la Información en la Educación y en la Sociedad. Una visión crítica. http://ties2012.eu

Secretaría de Comunicaciones y Transporte. (2019, 2 de abril). En México hay 74.3 millones de usuarios de internet y 18.3 millones de hogares con conexión a este servicio: ENDUTIH 2018. Gobierno de México. https://bit. ly/3hHIm1K

Secretaría de Econmía de México. (2018, 12 de enero). México Emprende. Economia. http://www.2006-2012.economia.gob.mx/mexico-emprende

Torres-Flórez, D. (2020). La generación de valor entre las personas y las organizaciones. Revista Gestión, Organizaciones y Negocios, 7(1), 4-8. https://doi. org/10.22579/23463910.211

Arenas-Martínez, E.C. (2018). Recursos TIC para la enseñanza y aprendizaje. Universidad Santander Panamá. http:// www.usantander.edu.pa 
Valencia, J. R. (1999). Facultad de Administración de UAP (1959-1999). Gaceta Histórica de la Benemérita Universidad Autónoma de Puebla, 2(10). https://bit. ly/2ELkGKm
Vivas, M. (2020, 26 de marzo). Ventajas y desventajas de las plataformas virtuales. Plataformas de educación virtual. http://plataformaseducvirtual.blogspot.com/p/v.html 